\title{
Prescribing Trends and Rationality of Fixed Dose Combinations in a Tertiary Care Hospital: An Observational Study
}

AMALU ROSE MATHEW, MINNU JOSEPH ${ }^{*}$, N. K. MEERA AND A. S. REEMA ${ }^{1}$ 
Department of Pharmacy Practice, Visveswarapura Institute of Pharmaceutical Sciences, ${ }^{1}$ Kempegowda Institute Of Medical Sciences, Bangalore-560 070, India

\section{Mathew et al.: Prescribing Trends and Rationality of FDCs}

This investigation aimed to review the prescribing trends of fixed dose combinations, to assess their rationality and inclusion in essential medicines list and national list of essential medicines. Medication charts of 1000 in-patients from general medicine department were reviewed for 12 months excluding casualty and ICUs. The data on morbidities and drugs prescribed were documented and assessed for fixed dose combinations prescribed, their inclusion in WHO essential medicines list and national list of essential medicines and approval by regulatory bodies such as US FDA and DCGI. Rationality of the prescribed fixed dose combinations were determined based on WHO guidelines. The drug-drug interactions with fixed dose combinations were analysed. Descriptive statistics was used to express results in numbers and percentages. Out of 1000 case sheets studied a total of 435 fixed dose combinations were prescribed, all by their brand names during hospitalization. Fixed dose combinations given for infectious diseases were $\mathbf{2 9 . 5 7} \%$ and for respiratory disorders $\mathbf{2 0 . 8 2} \%$. Those included in WHO essential medicines list 2017 were $11.72 \%$, while $10.57 \%$ were in the national list of essential medicines 2015 and $17.7 \%$ were approved by the US FDA, $56.78 \%$ by DCGI. Rational fixed dose combinations were $38.62 \%$ and $61.37 \%$ were irrational. In the discharge medication chart, miscellaneous agents $(19.72 \%)$ and drugs for infectious disorders $(15.80 \%)$ were the commonly prescribed fixed dose combinations. Among these $8.25 \%$ were listed in WHO essential medicines list 2017, $6.78 \%$ in the national list of essential medicines 2015 and $15.04 \%$ fixed dose combinations were approved by the US FDA, $53.98 \%$ by the DCGI. Rational combinations were $36.87 \%$ and $63.12 \%$ were irrational. Rationality in combining drugs as fixed dose combinations and their appropriate use can reduce pill burden, cost and improve patient adherence.

Key words: Fixed dose combinations, rationality, WHO guidelines, essential medicines list, national list of essential medicines

Fixed dose drug combinations (FDCs) are defined by the World Health Organization (WHO) as a combination of two or more active ingredients in a fixed ratio of doses and in a single dosage form ${ }^{[1]}$. Drugs from different pharmacological groups with complementary mechanism of action should be combined in FDCs. When they are combined in a single formulation, the safety, efficacy and bioavailability profiles of the established drugs change, and hence, FDCs are treated as new drugs ${ }^{[2]}$. Physicians prescribe a number of FDCs today in which majority of them are irrational.

FDCs are widely accepted when it offers justifiable advantages over the products with single active pharmaceutical ingredients (API ${ }^{[3]}$. Advantages of FDCs include better efficacy, reduced adverse drug reaction (ADR), provide broader spectrum of antibacterial activity, reduced complications, ease of administration and reduced polypharmacy. The use of combination drugs with fixed dose helps exhibit its effects with fewer pills or dose, thus improving the patient compliance. It may also reduce the cost and offer the poor patients a lower overall health care cost. The drugs in combination may provide a synergistic or

*Address for correspondence E-mail: minnujoseph8@gmail.com an additive effect. The use of antimicrobial FDCs like amoxicillin+clavulanic acid and ritonavir+lopinavir for the treatment of infectious diseases may slow or delay the attainment of antimicrobial resistance. There may be a decrease in side effects of individual drugs when these drugs are given in combination. However, there are certain disadvantages that are associated with the use of FDCs. These include potential bioavailability problems, difficulty in assessing ADR, emergence of resistance, increase in cost of therapy and poor patient compliance as they disagree to switch to FDCs. The dosing schedule of individual constituent of FDCs may differ and this may result in inflexible dosing regimen. There may be interaction among constituents of FDCs and this may result in adverse effects, particularly when drugs belonging to the same pharmacological class are used ${ }^{[4-6]}$. The increased number of prescriptions

This is an open access article distributed under the terms of the Creative Commons Attribution-NonCommercial-ShareAlike 3.0 License, which allows others to remix, tweak, and build upon the work non-commercially, as long as the author is credited and the new creations are licensed under the identical terms 
with FDCs is a matter of concern as it may develop irrational prescribing patterns in hospitals. It imposes unnecessary financial burden, increase the occurrence of ADR including allergy, hospitalization and ultimately reducing the quality of life. However, the advantages and disadvantages can mutually overlap ${ }^{[7]}$.

The WHO essential medicines list (EML) 2017 has 433 essential drugs, including 33 FDCs. Whereas, the national list of essential medicines (NLEM) 2015 includes 376 essential drugs, in which 22 are FDCs ${ }^{[8,9]}$. Nevertheless, the number of available FDCs in the Indian drug market is very large when compared to WHO EML and NLEM. There are more than 6000 FDCs in the market today. In view of this, this study was done to analyze the general medicine inpatient medication charts for the prescribing pattern of FDCs and their rationality.

A prospective observational study was conducted in the in-patient wards of general medicine department of a tertiary care teaching hospital, Bangalore, India. This study was carried out over a period of 12 mo from January to December 2018. The study was initiated after obtaining the approval from the VIPS Human Ethics Committee, Bangalore, India. (Ref. No:VIPS/ IEC 2017-02 dated 10-11-2017).

In-patient medication charts from the medicine department containing at least one FDC including supplements were analyzed irrespective of patient age, gender, disease diagnosed and route of administration. Medication charts with the FDCs from the casualty and intensive care units were excluded.

The in-patient case sheets were reviewed and the required data were recorded in a self-designed structured data collection form. Each patient case sheet was studied for patient's demographic data like age, gender, social habits, allergy status, duration of hospital stay, medical and medication history, current diagnosis, laboratory data, pharmacotherapy including FDCs, their brand name, generic name, dose, route, frequency and duration of treatment during the hospital stay; medications including FDCs in the discharge medication chart.

The data collected were analyzed for the number of FDCs, APIs in the FDC, pharmacological category, frequency and therapeutic duplication. The FDCs identified were assessed for their use as per WHO EML 2017 and NLEM 2015.

The rationality of the FDCs were assessed using a 7 point scale developed based on WHO guidelines which were, inclusion in WHO EML; NLEM; both; none. Approved by, USFDA, DCGI, banned. Appropriateness of the FDC, appropriate; inappropriate. Safety and efficacy of the FDC, safe; efficacious. Pharmacokinetics of FDCs, altered; unaltered. Mechanism of action, similar; complementary. Advantages, compliance; cost; dose.

A scoring of +1 is given for the positive findings and -1 for the negative findings with a total score of 13. Score of $\geq 7$ was considered rational and $\leq 6$ as irrational ${ }^{[1,10]}$. Any pharmacokinetic/pharmacodynamic interactions with APIs of FDC were identified and assessed for severity of interactions using available literature databases like Lexicomp, Stockley's drug interactions and www.drugs.com. Data were tabulated and analyzed in Microsoft Excel. Descriptive analytical statistics is used and the results were analyzed in terms of numbers and percentages.

Out of 1000 case sheets studied, the number of times FDCs were included in the inpatient medication charts and at the discharge were found to be 2377 and 1430 , respectively and an overall of 435 different FDC formulations. Majority of the cases contained more than one FDCs $(74 \%)$ and all the FDCs found were prescribed by their brand names. The study population included $57.8 \%$ male and $42.2 \%$ female with average ages of $49.92 \pm 16.79$ y and 49.90 $\pm 16.80 \mathrm{y}$, respectively. Those study patients who informed about alcohol consumption and smoking were $7.7 \%$ on assessing the social habits. The past medication history of the patients showed that the most common prescribed combinations were antidiabetics (52.34\%), antihypertensives $(31.54 \%)$ followed by antiplatelet agents $(9.39 \%)$, antiasthmatics/COPD preparations $(4.02 \%)$ and antiinfectives $(2.68 \%)$. Cardiovascular disorders, endocrine disorders followed by respiratory and hematological diseases were dominant morbidities. The anatomical classes of FDCs prescribed are depicted in Table 1. Those included in WHO EML and NLEM are depicted in fig. 1. The approval status of FDCs by statutory bodies like the US FDA and the DCGI is shown in fig. 2.

The most commonly prescribed antibacterial FDCs $(n=666)$ were cefoperazone + sulbactam $(37.68 \%)$ and cefoperazone+tazobactam (19.21\%). The repeated brands found were Inj. Stycef $(15.76 \%)$ and Inj. Phurox T (11.26 \%). Levosalbutamol+ipratropium bromide $(70.96 \%)$ and montelukast+levocetirizine $(17 \%)$ were the commonly found generic FDCs among the 341 antiasthmatics/COPD preparations. Neb. Duolin (70.96 \%) and Tab Montek LC (12.3\%) were 
TABLE 1: ANATOMICAL CLASSWISE FDC DISTRIBUTION

\begin{tabular}{lcc}
\hline Anatomical class & No. & $\%$ \\
\hline CVS & 272 & 11.443 \\
RS & 495 & 20.82457 \\
GI & 165 & 6.941523 \\
HEMATOLOGICAL & 48 & 2.019352 \\
HEPATIC & 110 & 4.627682 \\
CNS & 24 & 1.009676 \\
RENAL & 0 & 0 \\
PAIN/MS & 141 & 5.931847 \\
ENDOCRINE & 104 & 4.375263 \\
INFECTIONS & 703 & 29.57509 \\
OTHERS & 315 & 13.252 \\
& 2377 & 100 \\
\hline
\end{tabular}

CVS: Cardiovascular system, RS: Respiratory system, GI: Gastrointestinal, CNS: Central nervous system, MS: Musculoskeletal

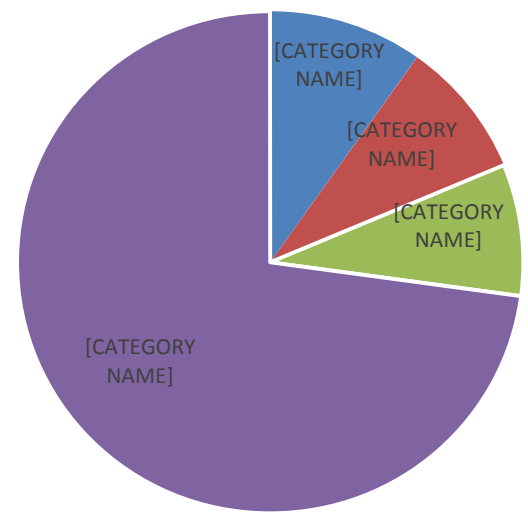

Fig. 1: Inclusion in WHO EML and NKEM

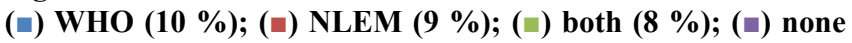
$(73 \%)$

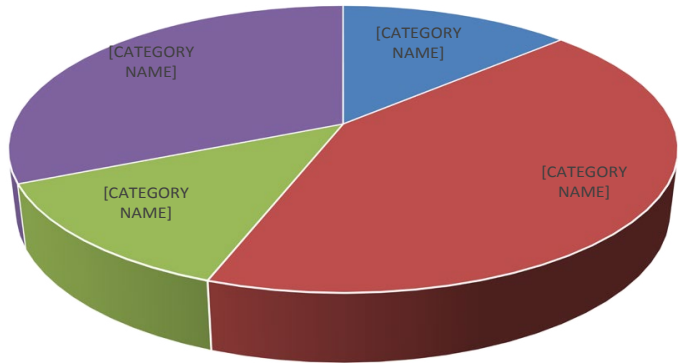

Fig. 2: Approval by US-FDA and DCGI

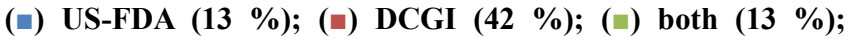
(घ) none (32 \%) the common brands. There were 289 supplements prescribed, in which, calcium carbonate+vitamin D3 $(10.72 \%)$ and vitamin B12+vitamin B6+nicotinamide $(5.53 \%)$ were the commonest generic combinations. Inj. Optineuron (24.56 \%) and Inj. Meconerve Forte (7.26 $\%$ ) were the frequently found brands. $38.62 \%$ rational and Among the FDCs found in the medication chart $38.62 \%$ were rational and $61.37 \%$ were irrational, a few examples are shown in Table 2. Major interactions observed were $3.95 \%$ while $30.29 \%$ were moderate and $9.29 \%$ minor drug interactions were observed and a few examples are shown in Table 3.

The discharge medication sheets of the study subjects were also analyzed in the similar way as of medication charts. The majority of the FDCs prescribed were as miscellaneous agents $(19.7 \%)$ followed by antiinfectives (15.8\%) and $14.19 \%$ for cardiovascular disorders and gastrointestinal disorders.

FDCs in the discharge medication list were assessed for their inclusion in WHO EML and NLEM and it was found that $8.25 \%$ were listed in WHO EML and $6.78 \%$ were found in NLEM. On assessing their approval status by US FDA and DCGI, majority of them were approved by DCGI $(53.98 \%)$ and $15.04 \%$ by USFDA however, $45.42 \%$ not included in both. The most prescribed FDCs in the discharge medication chart were also recorded. About 262 supplements were prescribed, e.g. combination of vitamins and minerals (Syp. Rd Vit $12.21 \%$, syrup Polybion $8 \%$ ), calcium with vitamin D3 (Tab. Shelcal HD $4.58 \%$ ). Out of the 218 antibacterials prescribed the commonly found combinations were cefpodoxime+clavulanic acid (48.16\%) and cefixime+lactobacillus $(21.1 \%)$. The frequent brands prescribed were Tab. Cefozyt CV (28.4\%) and T. SedcefLB (21.1\%) while $36.87 \%$ of the combinations were rational and $63.12 \%$ were found to be irrational.

Drug-drug interactions of the FDCs in the discharge medication chart with other prescribed drugs as well as within the combination were assessed and there

TABLE 2: RATIONALITY ASSESSMENT

\begin{tabular}{lccccc}
\hline PHARMACOLOGICAL CLASS & RATIONAL & $\%$ & IRRATIONAL & $\%$ & TOTAL \\
\hline Antihypertensives & 36 & 69.23077 & 16 & 30.76923 & 52 \\
Antiplatelets/thrombolytics & 11 & 44 & 14 & 56 & 25 \\
Cough and cold preparations & 6 & 16.21622 & 31 & 83.78378 & 37 \\
CNS drugs & 12 & 75 & 4 & 25 & 16 \\
Pain/musculoskeletal drugs & 7 & 21.875 & 25 & 78.125 & 32 \\
Antidiabetics & 7 & 20 & 28 & 80 & 35 \\
Antibacterials & 50 & 68.49315 & 23 & 31.50685 & 73 \\
Supplements & 7 & 10.44776 & 60 & 89.55224 & 67 \\
\hline
\end{tabular}

CNS: Central nervous system 
TABLE 3: EXAMPLES OF DDIS

\begin{tabular}{lc}
\hline SEVERITY & EXAMPLES \\
\hline Major & Spironolactone, ramipril \\
& Clarithromycin, atorvastatin \\
Moderate & Metformin, glimepiride \\
& Aspirin, clopidogrel \\
Minor & Aspirin, pantoprazole \\
& Piperacillin, clarithromycin \\
\hline
\end{tabular}

were 29 (2.02\%) major, 459 (32.09\%) moderate and $151(10.55 \%)$ minor interactions.

The current study was carried out to assess the trends in usage of FDCs by the clinicians and assessed for their rationality. The medication charts including the discharge medications of inpatients from general medicine wards were assessed for a period of one year. Seventy four percent of the medication charts included at least one FDC and prescribed by brand names. About 435 different FDCs were identified, out of which 11.72 and $10.57 \%$ of the prescribed FDCs were found to be included in WHO EML and NLEM, respectively. Approved FDCs were 17.70 and $56.78 \%$ by the USFDA and the DCGI, respectively. It was identified that FDCs used to treat infections were maximum followed by drug combinations used for treatment of conditions of respiratory system, cardiovascular system. On assessing the combination of drugs based on their pharmacological class, it was found that $69 \%$ of antihypertensives were rational while $31 \%$ were irrational. Similarly $68 \%$ of antibacterials were rational while $32 \%$ were irrational combinations. Cefoperazone+sulbactam (Inj. Srycef) and cefoperazone+tazobactam (Inj. Phurox T) were commonly preferred antibacterial FDCs. Neb. Duolin and Tab. Montek LC were the common brands of combinations of levosalbutamol+ipratropium bromide and montelukast+levocetirizine. Supplements like combinations of calcium+vitamin D3 and multivitamin preparations were prescribed of which $10 \%$ were rational and the rest $90 \%$ irrational.

Appendix VI of schedule Y of Drugs and Cosmetics Act 1945 provides the specific requirements for approval of FDCs marketed in India ${ }^{[11]}$. The most common FDC preparations are prescribed for a broad spectrum of conditions and the combinations comprise of vitamins/ supplements, antibacterials, preparations for cold and cough, antacids. The rationality of a major number of FDCs available in the pharmacies is highly questionable. WHO provides examples of drug combinations like sulfamethoxazole+trimethoprim, antitubercular drug combinations like rifampicin+isoniazid+ethambutol, combinations of levodopa+carbidopa for treatment of parkinsonism, which are known to be rational ${ }^{[12]}$. Many researchers have expressed similar findings with respect to the approval and rationality of FDCs ${ }^{[1,13]}$.

In the present study, several irrational combinations were found and to quote a few, ambroxol+guaifenesin + menthol+terbutaline and ambroxol+guaifenesin+terbutaline under cough and cold preparations, pregabalin+vitamin B6+folic acid+alpha lipoic acid+vitamin B12 and thiamine+pyridoxine+riboflavin+vitamin $\mathrm{B} 12+$ nicotinamide $+\mathrm{D}$ panthenol under the category of supplements, metformin+glimepiride and metformin+glimepiride+voglibose in antidiabetics were among those. In the case of cough and cold preparations many of the constituents of the preparations may not be required or those might be banned ingredients like phenylpropanolamine. According to DCGI, the FDC including vitamins B1, B6, and B12 in a single preparation provides no additional therapeutic benefit over individual vitamins ${ }^{[14,15]}$.

As mentioned earlier, the therapy with FDCs reduce the polypharmacy or pill burden, which in turn can improve patient compliance. However, the rationality and justification of their uses always raises doubt and it can lead to controversial usage of drugs.

Most commonly, the clinicians obtain information from the medical representatives apart from obtaining the information through peer group, resources like MIMS, CIMS, and continuing medical education programs. Insufficient or often biased information can lead to inappropriateness in the use of drugs. Strengthening of the regulatory guidelines, provision of continued updated unbiased information about the drug products and their safety should help in minimizing the inappropriate and irrational use of drugs.

Rational combination of drugs to formulate FDCs and the appropriate use of FDCs can definitely improve adherence to the therapy, safety, and reduce the cost of therapy. However, efforts to increase awareness regarding the correct use of FDCs should be a constant objective for the pharmacists.

\section{Conflict of interest:}

There are no conflicts of interest.

\section{Acknowledgements:}

The authors thank all the staffs in the general medicine ward of KIMS hospital and RC for their cooperation. 


\section{REFERENCES}

1. Guidelines for registration of fixed dose combination medicinal products. WHO Technical report series number 929. Geneva: World Health Organization; 2005.142 report number 39.

2. Rayasam SP, Dudhgaonkar SS, Dakhale GN, Hire RC, Deshmukh PS, Gaikwad NN. The irrational fixed dose combinations in the Indian drug market: an evaluation of prescribing pattern using WHO guidelines. Int J Basic Clin Pharmacol 2013;2(4):452-57.

3. Prajapati K, Shah S Desai M. Critical analysis of cardiovascular and central nervous system fixed dose combinations available in Indian Market. JCDR 2016;10(12):36-39.

4. Dahiya A. Fixed dose drug combinations in India-Is there enough scientific evidence. J Macrotrends Health Med 2014;2(1):85-94

5. Najmi A, Aiman U. A critical appraisal of rational and irrational fixed drug combinations. Asian J Pharm Sci Technol 2015;5(4):335-37.

6. Vijayakumar TM, Jayaram J, Cheekireddy VM, Himaja D, Teja YD, Narayanasamy D. Safety, efficacy and bioavailability of fixed dose combinations in Type 2 Diabetes Mellitus: A systematic updated review. Curr Ther Res Clin Exp 2017;84:49.

7. Balasubramanian J, Radhika N, Badarinath AV. The crave of fixed dose combination in Indian market. Asian J Pharm Clin Res 2014;7(4):106-10.

8. $20^{\text {th }}$ WHO Model List of Essential Medicines, 2017. Available from: http://www.who.int/medicines/publications/ essentialmedicines/en/

9. NLEM 2015. Available from: http://apps.who.int/ medicinedocs/en/m/abstract/Js23088en/

10. Pradhan S, Panda A, Sahu S, Behera JP. An evaluation of prevalence and prescribing patterns of rational and irrational fixed dose combinations (FDCs): a hospital based study. Int J Med Sci Public Health 2017;6(1):58-62.

11. Guidance for Industry on fixed dose combinations (FDCs). Available from: http://www.cdsco.nic.in/writerdata/FDC.

12. Gautam CS, Saha L. Fixed dose drug combinations (FDCs): Rational or irrational: A view point. Br J Clin Pharmacol 2008;65(5):795-6.

13. $19^{\text {th }}$ WHO Model List of Essential Medicines, 2015. Available from: http://www.who.int/medicines/publications/ pharmacopoeia.

14. Dawson KP, Fergusson DM. Effects of oral theophylline and oral salbutamol in the treatment of asthma. Arch Dis Child 1982;57(9):674-6.

15. Drugs banned in India. Available from: http://www.cdsco.nic. in. 